\title{
Promoting Local Culture and Enriching Airport Experiences through Interactive Storytelling
}

\author{
Alisa Burova ${ }^{1}$, Chelsea Kelling ${ }^{1}$, Tuuli Keskinen ${ }^{1}$, Jaakko Hakulinen ${ }^{1}$, Pekka Kallioniemi ${ }^{1}$, \\ Heli Väätäjä ${ }^{2}$, Markku Turunen ${ }^{1}$ \\ ${ }^{1}$ Tampere Unit for Computer-Human Interaction (TAUCHI), Faculty of Communication Sciences \\ Tampere University \\ Tampere, Finland \\ alissa.burova@tuni.fi; chelsea.kelling@tuni.fi; tuuli.keskinen@tuni.fi; jaakko.hakulinen@tuni.fi; \\ pekka.kallioniemi@tuni.fi; markku.turunen@tuni.fi \\ 2 Lapland University of Applied Science \\ Rovaniemi, Finland \\ heli.vaataja@lapinamk.fi
}

\begin{abstract}
Experiences in airports may shape future travel plans and contribute to tourism destination development. However, a chaotic environment and time-consuming procedural routines in airports may result in negative associations towards the host country and its culture. Despite the existence of assistive airport applications, little attention is given to facilitating travelers' engagement with cultural exploration.

This paper introduces a concept of interactive personalized storytelling that provides both a cultural learning adventure and connection to local retailing. Our application generates an imaginative Finnish storyline unique to every user to guide them through local shops in the airport. A field evaluation was conducted with 15 travelers of different nationalities. Travelers perceived the interactive storytelling experience as an interesting and unique way to spend waiting time at the airport while increasing cultural exposure. Moreover, we found this method to be effective in persuading travelers to explore local products at the airport. Further, our results give insight to designing storytelling applications for large public places.
\end{abstract}

\section{CCS CONCEPTS}

CCS $\rightarrow$ Human-centered computing $\rightarrow$ Human computer interaction $(\mathrm{HCI}) \rightarrow \mathrm{HCI}$ design and evaluation methods $\rightarrow$ Field studies

\section{KEYWORDS}

Digital storytelling, Airport experience, Mobile application, Field study.

\section{INTRODUCTION}

Airports facilitate global mobility, and thus, play a major role in the development of the tourism industry. Airports all over the globe have adopted a customer-oriented business model and structure their operations to manage and positively influence travelers' airport experience [18]. In addition to stimulating purchasing intentions, a positive airport experience may influence the future traveling plan creation [15] and support destination development [17]. Hence, it is equally important to shape a pleasant airport experience and link the airport activities to cultural exploration, thus expressing and distinguishing the uniqueness of local culture. Yet, the typical airport experience can hardly be described as "pleasant", as a chaotic airport environment triggers anxiety and other negative emotions [3].

Shopping is certainly one of the primary activities at the airport used to kill time and reduce anxiety [11]. Despite airports providing the means of illustrating shopping offers, they rarely draw relations to the local culture or persuade shopping intentions with little else than discounts or special offers. Further, mobile applications, a viable technology for the airport environment [13] to provide assistance in airport procedures and navigation [22, 23], are still rarely utilized to facilitate meaningful and cultural time spending. In particular, storytelling 
and gamification, which are widely used in the tourism industry to increase interest in the culture, have earned less attention from airport facilitators.

We fulfilled this gap by exploring how an interactive storytelling approach supported by gamification elements influence overall airport experiences, tourism destination development, and travelers' shopping intentions. Hence, we formulated the following research questions:

RQ1 - How can an interactive storytelling approach be utilized to highlight the culture of the destination and personally connect travelers to local shop offers?

RQ2 - What are the possible effects of the solution on overall airport experience and shopping intentions?

To address these questions, we developed an application, called The Finnish You, which generates a personal and unique storyline to guide travelers through local brands and shops in the airport. To strengthen our findings, and in contrast to a preceding study held in lab settings [5], we extracted data from a challenging real-world context and observed the usage of such an application in a field study with 15 travelers of different nationalities. Our findings indicate that interactive storytelling is a promising strategy to present local culture and lifestyle in an easy-to-receive manner, and, at the same time, persuade travelers to visit local shops and explore shopping possibilities.

\section{RELATED WORK}

\subsection{Airport experience}

The role of airports has shifted from being transition spaces [8] to being massive experiencescapes consisting of endless commercial attractions, such as shopping, food and beverage, and other services [5]. Despite airports being primarily associated with excitement, by virtue of expected change from everyday routine [8], travelers also face negative feelings, e.g., stress, anxiety, and boredom, as a consequence of exhausting airport procedures and constant waiting [3]. Although airports are seen as a destination ambassador [17], and not a destination itself [10], all airport activities together form an airport experience, which is perceived as an integral part of the tourism experience [17]. Hence, forming a pleasant airport experience is critical to influencing tourism destination development and, potentially, future travel plan creation [15]. Wattanacharoensil et al. [18], for instance, described airports as experience providers and facilitators and further suggested connecting travelers with a destination by incorporating a "sense of place" via cultural activities and artefacts, and by promoting social interactions and co-creation processes. This is especially relevant for newcomers and transfer passengers, who experience the airport atmosphere in a similar way as visiting a tourist destination [10].

Further, attracting passengers to partake in commercial activities and encouraging money spending in local shops is undoubtedly among the main goals of airport officials, as retailing revenues account to be more than half of total airport revenue [3]. Therefore, airports perceive travelers as their direct customers [18] and constantly develop new strategies to attract them to partake in consumer experiences. Recent research in shopping motivation revealed that travelers' activities are influenced by environmental and cultural motivators [11], and thus, a shopping environment that facilitates meaningful experiences is more valuable for travelers than an environment that only fits their shopping tasks [6].

\subsection{Digital solutions in tourism}

With the rapid maturing of mobile technologies, it has become possible to facilitate and enhance both touristic and airport experiences by using personal devices. In the tourism sector, various solutions have been introduced to engage travelers in a destination and cultural heritage exploration $[2,4,7,12,16]$ by utilizing digital storytelling or gamification, among other strategies. For instance, digital storytelling, which guides visitors to a historical site, was found to enhance the identity of the destination by affecting travelers emotionally and increasing the receptiveness and precision of conveyed information [12]. In addition, stories and narratives are efficient in promoting products, services, or locations [14], in virtue of their proven ability to influence consuming intentions [1] and shaping the consumption experiences [21].

Likewise, gamification is applied to attract tourists' attention to cultural heritage [4], raise local brand awareness [20], and thus, improve tourist experiences by stimulating fun, curiosity, social interactions, and the feeling of challenge [19]. For example, Ardito et al. [2] presented the excursion-game technique to make archeological visits more exciting, while Corrêa and Kitano [7] discussed the benefits of using gamification elements to persuade tourists to explore the country and distinguish its culture. Based on the success of digital storytelling and gamification, we aimed to utilize these elements to generate a unique story tailored to the users' personalities while embedding shopping options into the story to connect elements of fun, curiosity, and challenge $[19,20]$ to the retail experience, thus improving travelers' overall airport experience.

\section{THE FINNISH YOU: INTERACTIVE STORYTELLING APPLICATION}

To explore how the concept of interactive storytelling may affect passenger experience and, in particular, shopping intentions, we developed a web-based mobile application, The Finnish You ${ }^{1}$. The central idea of the application is to tell a unique story about the user's life as if they had been born in Finland. The application generates a personalized storyline to present culture-related information and simultaneously guides travelers through local shops at the airport while recommending products to be purchased as a part of the story.

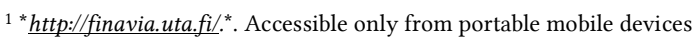


Hi! My name is Kaarina. I am just like you, but born in Finland. Kaarina translates from Finnish as 'pure'. I live in Espoo, the second largest city nearby Helsinki. Although relatively

highly populated, it has large amounts of natural wilderness: the city has a total of 71 lakes and a long coastline on the Gulf of Finland.

I'm working in Fazer cafe. I love to see happy children faces, when they try a cake cooked by me.

I have a dog of a Finnish lapphund breed, named Turre.

I hate sitting home, so I usually spend my free time outside. Luckily, I have a ski trace right in front of my house.

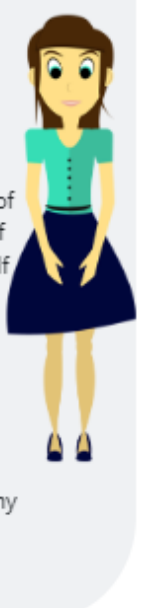

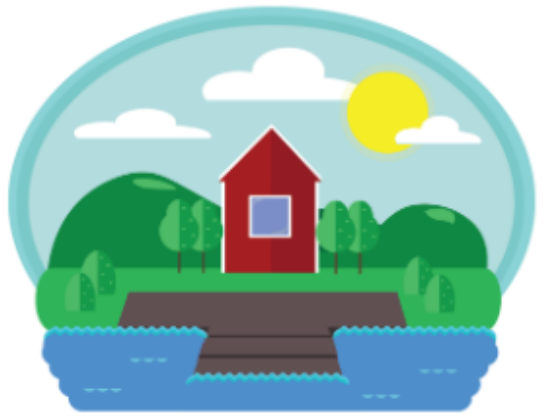

This Friday, my Dad will have Vappu celebrations in family house. To prepare, I need to do some shopping.
Hi! My name is Miika. I am your Finnish version. Miika refers to 'who looks like God'.

I live in Utsjoki, the northmost place in Finland.

I'm working in Finnish forest industry company Metsa. The job is hard, but I like it, because I spend working hours on nature.

I have a cat of a European shorthair breed, named Mirre.

I am a big hockey fan, so whenever there is an interesting game, I invite my best friend to watch the match on my big plasma and drink few beers together.

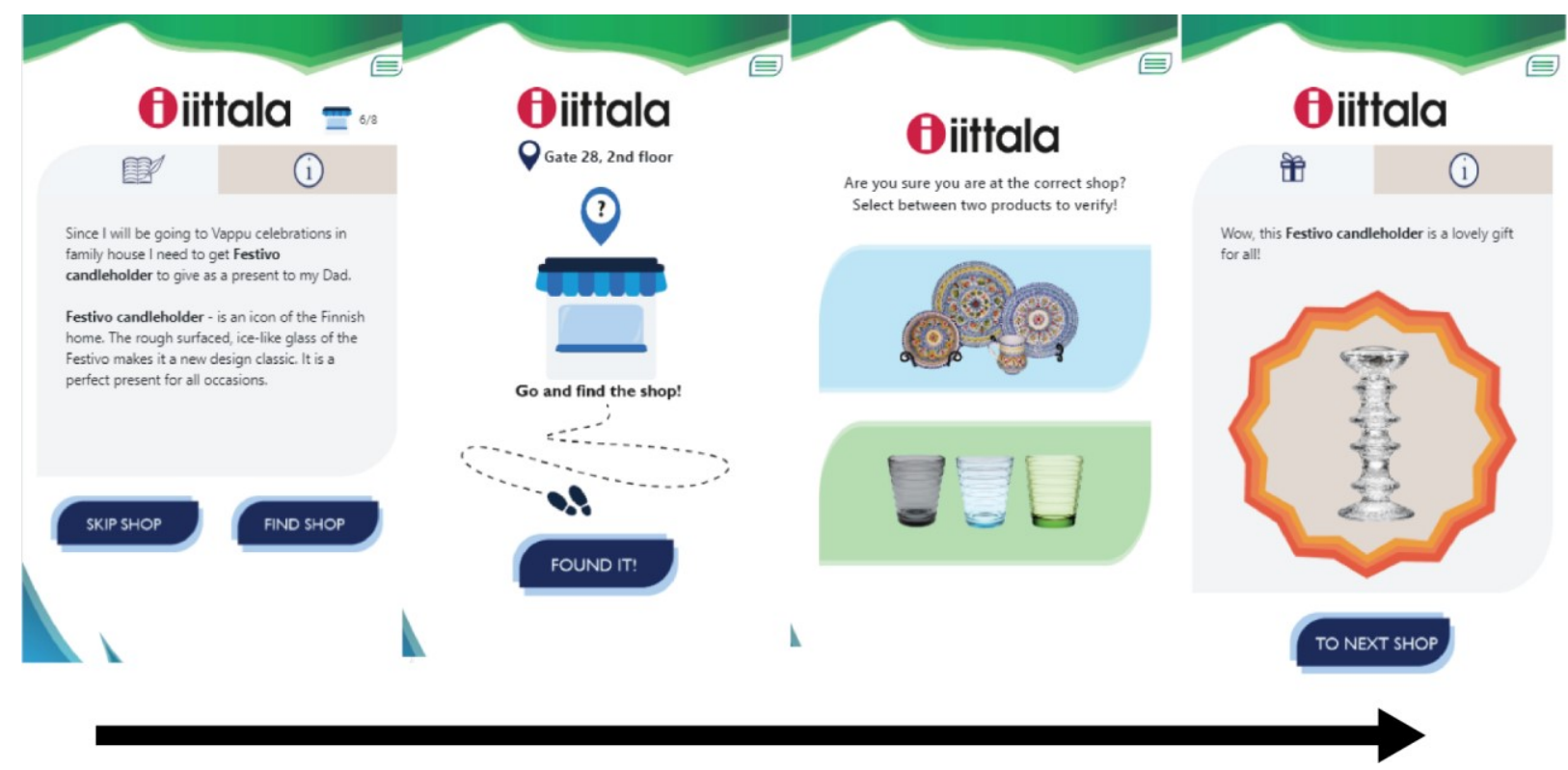

Fig 1. Two alternative introduction stories (top left and right) and the invitation to the virtual event (top center), and the process of finding the Finnish shop, Iittala (bottom).

The application concept and functionality are introduced as onboarding pages before the story begins. The application is composed in a flat design style, using graphics, user interface elements, and colors to aesthetically draw the connection to the Finnish culture and create a feeling of fantasy [5]. To stimulate fun and adventurous experiences with the application, it utilizes supportive gamification elements, such as avatars, progress bars in the form of a Finnish map and shop counter, the challenge of locating the shops themselves and finally, rewards for physically visiting the shops.

The personalized storyline is built based on the user's responses to background questions prompted after the onboarding screens, including age, gender and personal preferences in the form of quiz-like alternatives where users choose which they like best for climate (cold/warm), place of living (city/village) and lifestyle (active/calm). The story consists of three parts presented as text with illustrations. The first part, an introduction, narrates the user's Finnish lifestyle (two introduction examples are presented in the Fig. 1, top left and right) and invites them to take part in a virtual event that triggers a virtual shopping adventure (Fig. 1, top middle).

The story segments are generated with context-free grammars [9], one for each part. They define text fragments and how they can be combined. There are in total 494 rules in the grammars and many of these contain alternative phrasings, of which one is randomly selected. For example, the start of the story in Fig. 1 is formed by a rule "bioIntro" with child nodes "HiNameIs", "name", "genderNode", "period" and "likeYou". Of these, "likeYou" can be expanded to two literals "I am just like you, but born in Finland" or "I am your Finnish version." In 
addition, a global feature structure is maintained during story generation; rules can set feature values, and requirements of rules applied later are compared to them. In our example, "genderNode" matches feature "gender" as defined by the user to "male", "female", or "neutral" and this feature then controls the selection of a person's name and avatar. Overall, the features are used to ensure the story is coherent both within and across the three parts.

The second part consists of persuasion statements to randomly invite the user to one of 8 shops and advertises the product and the shop with a brief but sophisticated narration. The users have a choice to visit or skip the suggested shop as well as view a map of the shops and select a shop manually. If the user selects to visit the shop, the application provides tips on where to find the shop without straightforward guidance. This approach was selected because the Helsinki airport is comparatively small in size and easy to navigate with only the gate and floor numbers. Further, the goal of this approach is to persuade the users to look around and explore all shopping possibilities while finding the correct shop. When the user correctly locates the shop, the application asks for verification. In the verification section, the application shows two products on the screen. The user should then explore the shop items around them and select one of the two products on the screen that looks most similar to what the shop has to offer. If the selection is correct, the user gets a reward and continues the adventure. The process of locating the shop is demonstrated in Fig.1, bottom.

The third part of the journey is a summarized story, which describes all of the products "purchased" virtually and presents more cultural information by describing the virtual event and cultural specifics of it, e.g. how Finnish people would spend a Vappu (May Day) celebration in their summer cottage. The summary can then be shared to social networks or uploaded as a pdf file to the user's portable device for later viewing.

\section{FIELD STUDY AT HELSINKI AIRPORT}

The qualitative field study at the Helsinki airport aimed to observe the usage of the application in a real context with actual tourists. Due to logistic and security restrictions, we (three researchers with native English, Chinese and Russian language skills) agreed with airport officials to arrange a one-day study in a terminal area under the supervision of an airport representative.

\subsection{Methodology}

The focus of the study was to collect subjective opinions about the interactive storytelling approach and observe its possible effects on shopping behavior. To achieve this, we used an observation form (filled by the researchers) to note the patterns of interactions between the users, the application, and the environment. We also used structured interviews to obtain insights on the application's strongest and weakest points and a brief 5-point scale questionnaire on paper (adapted from QUIS [24] and USE [25] questionnaires) to collect user experiences in a quantitative and comparable form. The testing procedure consisted of a brief introduction to the study and then free-form application usage. Each session was held with one participant at a time or a pair of two participants traveling together. Each participant used a Samsung Galaxy S7 (with Android 7.0) for the duration of the study, which on average took around 45 minutes. The sessions were audio-recorded with participants' written consent. A researcher accompanied the participants in the shop exploration, assisted with the application usage when required and ended the application usage after the 3rd shop visit with a task to open the summary of their adventure.

A total of 15 participants were recruited at the terminal area (9 male, 6 female), aged 19 to $47(m=27)$. Eight of the participants were Chinese by nationality, four were Russian, and the remaining were French, Israeli, and Nepalese. The application itself and the questionnaire were in English, but the communication with the participants took place in English, Chinese, or Russian as applicable. All the interview data was then further translated into English for analysis.

\subsection{Results}

\subsubsection{Interview and Questionnaire}

As shown in Fig. 2, the overall reaction to the application was positive. The majority (80\%) of the participants found the application attractive; eight participants (53.3\%) chose the positive extreme. The application was also perceived as easy to use and creative (twelve participants (80\%) on the positive side). Participants commented, for example:

"I liked the concept because it is not a typical, but funny and cool way to go through the shops." (P1, aged 19, female)

"I liked that it tells the story about the country and the shops. We are in Finland now and it's interesting to know how people live here." (P2, aged 30 , female)

Only 2 participants (13.3\%) reported that the application was somewhat difficult to use and only one (6.6\%) that it was somewhat frustrating. However, some participants explained that due to a high cognitive load, they were not able to fully concentrate and skipped the text from the on-boarding pages that detailed the description and purpose of the application.

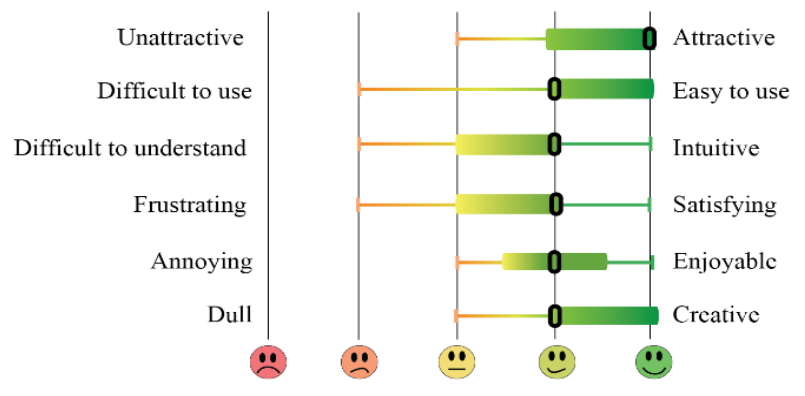

Fig 2. Participants' overall reactions to the application. 
Further, two participants $(13,3 \%)$ expected to see a typical shopping application with a list of products, rather than a personalized storyline. Four participants (26.6\%) expressed interest in discounts and special offers, and therefore, the concept of the application did not fit their needs; one of them also did not see the point of sharing personal information, such as age and gender, for shopping purposes.

The personalized storyline was perceived differently from participant to participant. About half of the participants (53.3\%) agreed or strongly agreed that it was interesting to read about the Finnish version of themselves. Ten participants $(66.6 \%)$ agreed or strongly agreed that the summary was interesting, and six $(40 \%)$ further agreed or strongly agreed that they could relate to the story. Similarly, as we identified the persuasive effect towards exploring the local shops and products, some participants did notice the effect on their purchasing intentions. The application successfully made participants discover previously unknown brands and visit the shops to observe what they could purchase.

Participants also showed interest in discovering the products recommended by the application, as they were touching the products, trying them on, and asking questions. Participants said, for example:

“...if it (the recommended product) fits my needs, I think this service would stimulate my desire to purchase." (P3, aged 22, female)

"There are some shops that would not attract my attention, so with the app, I noticed them [...]. It set my attitude to explore more.” (P4, aged 45, male)

\subsubsection{Observations}

Fig. 3 presents the results on how well the application's textual information was able to engage the participants. The introduction story appeared to be more attractive and engaging to read than the summary story. Nine participants (60\%) read the introduction story with interest; some of them asked questions about the story or about Finnish culture, while only four (26.6\%) read the summary story fully. We also found that eight (53.3\%) participants read the persuasion statements and product descriptions, while seven $(46.6 \%)$ only scanned them with their eyes. Considering the effect on shopping behavior, we found that the application persuaded all of the participants to walk at least near to the shops. Although five participants (33.3\%) made the verification without entering the shop, four (26.6\%) did visit the shop for verification, two (13.3\%) to explore the products, and even four (26.6\%) visited the shop for both verifications and exploring the products.

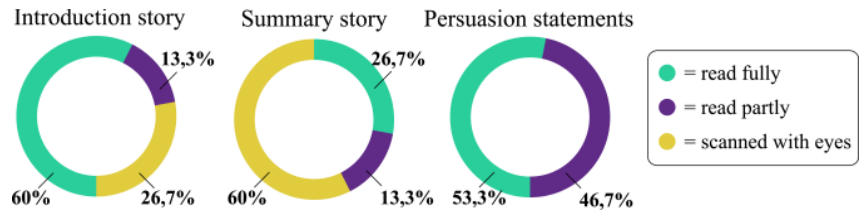

Fig. 3. Engagement with various textual information.
The participants traveling in pairs discussed and shared their storylines with each other and cooperated to select the shop they wanted to visit; however, they verified the shop visit on only one device, which led to the loss of the route history and incomplete summary stories of the adventure.

\section{DISCUSSION}

In summary, the results indeed demonstrate the potential of utilizing interactive storytelling in the airport context to facilitate a cultural learning experience. Further, our results suggest that a personalized storytelling approach can be utilized to fill waiting time when traveling and could successfully facilitate cultural adventures in other public touristic environments, such as ferry terminals, railway and bus stations, shopping malls, urban spaces, and natural parks. As for RQ1, we identified that an automatically generated personal storyline is indeed an appealing approach to present culture-related information and to connect travelers to local shop offers. Considering the uniqueness of the story for each user, this type of application may be advantageous to engage groups of tourists and thus, increase social interaction with a co-creation process.

As for RQ2, the qualitative findings reveal that people who seek entertaining ways to spend their time enjoyed the storytelling experience. The application enhanced their airport experience, introducing the local culture in a unique manner and filling airport waiting time with meaningful and fun activity. Moreover, there is a preliminary indication that such services may further spark travelers' curiosity in the destination by explaining the value of local products. Participants who found the storyline to be captivating, indeed explored the shopping possibilities at the recommended stores. Thus, our findings suggest that in addition to enhancing the "sense of place", the story generation technique also stimulates shopping intentions by creating a virtual atmosphere and emphasizing the uniqueness of local offers.

\subsection{Guidelines for a storytelling application for touristic public spaces}

Based on the findings from the field study, we extended the list of guidelines for interactive storytelling from a prior study that was conducted in a lab setting [5]. The preceding study guidelines contributed to enhancing the story segment itself, e.g., utilizing various methods of storytelling, adding playful activities, and improving the personalization and customization. In contrast, here we formulate guidelines from both the perspective of usability and of cultural and retailing promotion in the context of an airport. Presented guidelines may be also generalized for other touristic public spaces similar to the airport environment.

1. Clarity of the value proposition. Based on our results, utilizing the on-boarding pages as the method to communicate the value of the application seems to be inefficient in the airport context due to high cognitive load. Some of the users misinterpreted the idea of a cultural storyline, expecting only shopping related information. Therefore, the idea and value of 
using the application, e.g., time killing, cultural exploration, and personalized storyline, should be stated clearly and distinctly. The solution might be to provide an interactive description of the application, which could allow further redirection to various airport services according to user-defined needs.

2. Enabling context-awareness. Despite the promising results, the storytelling approach in its current implementation attracts a narrow group of travelers. For instance, people with a primary goal to shop efficiently might perceive the relatively long story as an unnecessary feature. Thus, such services should be adaptive and responsive to the needs of different user groups and support various application scenarios. For example, the qualitative results introduce users' demand for so-called sales tours, which would narratively guide users to the products with reduced prices.

3. Transparent instructions. Based on our results, delivering all instructions at the start of the application is a disadvantageous approach. Due to the chaotic airport environment, the application functionality should be provided on the go, e.g., displaying instructions step-by-step and only when the new features are introduced.

4. Facilitating cultural exploration. The majority of the participants were interested in getting more in-depth information about the Finnish lifestyle and also expressed the desire to feel the experience for themselves and visit Finland in the future. Providing links to external resources could support further information retrieval and enhance the aspect of cultural exploration, thus attracting travelers to visiting a country in the future.

5. Persuading shopping intentions. Although some participants did not fully relate to the Finnish persona, they still showed interest in exploring their story and visiting local shops to discover national products. To increase the persuasion effect, we identified the need to accumulate interactive and tangible experiences with the product suggestions made by the application. To enable such experiences, the application should be designed in tight cooperation with airport facility providers.

In addition to providing insight on how interactive storytelling can be utilized and enjoyed, this study revealed the challenges of arranging a field study at the airport, i.e. a public space with high security. To make the most of the time allowed in the airport, the three researchers conducted testing sessions separately. The nature of the study required the researchers to follow the participants in their adventure and thus, were spread throughout the terminal area. The delegated airport representative, who was tasked with the supervision of the study, could not follow all three at the same time and thus, the testing was interrupted by the security of the airport. All 15 participants did complete the testing procedure; however, the targeted amount of participants was not reached. Hence, based on our experience, when testing in a public space with high security we suggest to meticulously coordinate all study activities with representatives beforehand and agree on all procedures from both parties.

\subsection{Limitation and Future Work}

The contribution of this paper lies in the qualitative exploration of interactive storytelling in the context of a small-scale airport. We found that a personalized storyline, unique to every user, is a viable solution to illustrate culture-related information and stimulate shopping interest and thus, grants need for further research.

The main limitation of the study lies in the relatively small public space that was used for testing purposes, as well as the small number of participants with low background/demographic variance, which made it difficult to determine any usage patterns in relation to culture, gender or age. Hence, experiments with more participants could look into the potential of storytelling in more detail, explore culturally related differences in how storytelling should be applied and how the storytelling approach generalizes to people of different ages. From another perspective, the means of enhancing and fully utilizing the personalization of interactive storylines could be based on factors such as travel purpose, amount of available waiting time and other travelling habits, and should be further investigated to examine how to most effectively deliver truly engaging touristic experiences. In addition to user characteristics, contextual factors that could influence story creation and application design (e.g. size of the public space, season, time of the day, or location climate), should also be studied further. In large-scale public spaces, for instance, navigation to the shops should be designed in a more explicit manner, e.g. based on more specific guidance such as an augmented reality path overlay. Further, the cooperative use of personalized storytelling applications could be researched to target tourists traveling in groups and assist companies that are interested in increasing foot traffic to their shops.

\section{CONCLUSION}

This study explored how an interactive storytelling approach may be applied in the context of an airport and how it effects airport experiences and shopping behaviors. We deployed the The Finnish You application, which generates a personal storyline to present culture-related information and guides travelers through the local shops, and conducted a field study with 15 participants of different nationalities at the terminal area of the Helsinki airport. Our study highlights the potential of storytelling generation techniques and shows the benefits of presenting cultural information in the form of a personal story to travelers who are searching for entertainment at the airport. Further, such an application may contribute to destination development and stimulate purchasing intentions by augmenting the shopping environment and highlighting the uniqueness of local products. Therefore, we believe our results and extended storytelling design guidelines can be utilized by others in the design and research of services for public touristic environments.

\section{ACKNOWLEDGMENTS}

This research was done in the VIRJOX project, funded by Tekes the Finnish Funding Agency for Innovation (later Business Finland). We thank Finavia for their close cooperation and granted access to the airport terminal area to conduct the field study. 


\section{REFERENCES}

[1] Ali Ekber Akgün, Halit Keskin, Hayat Ayar, and Ebru Erdoğan. 2015. The influence of storytelling approach in travel writings on readers' empathy and travel intentions Peer-review under the responsibility of the International Strategic Management Conference. Procedia - Social and Behavioral Sciences 207: 577-586. https://doi.org/10.1016/j.sbspro.2015.10.129

[2] Carmelo Ardito, Paolo Buono, Maria Francesca Costabile, and Rosa Lanzilotti. 2009. Enabling Interactive Exploration of Cultural Heritage: An Experience of Designing Systems for Mobile Devices. 79-86. https://doi.org/10.1007/s12130-009-9079-7

[3] Bodil Stilling Blichfeldt, Aurimas Pumputis, and Kiya Ebba. 2017. Using, spending, wasting and killing time in airports. International Journal of Culture, Tourism, and Hospitality Research 11, 3: 392-405. https://doi.org/10.1108/IJCTHR-05-2016-0045

[4] Armir Bujari, Matteo Ciman, Ombretta Gaggi, and Claudio E. Palazzi. 2017. Using gamification to discover cultural heritage locations from geo-tagged photos. Personal and Ubiquitous Computing 21, 2: 235-252. https://doi.org/10.1007/s00779-016-0989-6

[5] Alisa Burova, Chelsea Kelling, Jaakko Hakulinen, Pekka Kallioniemi, Tuuli Keskinen, Markku Turunen, and Heli Väätäjä. 2018. The Finnish You: an Interactive Storytelling Application for an Airport Environment. In Proceedings of the 22nd International Academic Mindtrek Conference (Mindtrek '18). ACM, New York, NY, USA, 182-191. DOI https://doi.org/10.1145/3275116.3275142Yi

[6] Shih Chung. 2015. Hedonic and utilitarian shopping values in airport shopping behavior. Journal of Air Transport Management 49: 28-34. https://doi.org/10.1016/j.jairtraman.2015.07.003

[7] Cynthia Corrêa and Camila Kitano. 2015. Gamification in Tourism: Analysis of Brazil Quest Game 1 Introduction and Theoretical Background. ENTER 2015 Conference on Information and Communication Technologies in Tourism 8, 3: 1-2. https://doi.org/10.19080/PBSIJ.2018.08.555740

[8] Mark. Gottdiener. 2001. Life in the air: surviving the new culture of air travel. Rowman \& Littlefield. Retrieved June 28, 2018 from https://books.google.fi/books?hl=en\&lr=\&id=ML9kWO_FEq0C\&oi=fnd\&pg= PA1\&dq=Life+in+the+Air:+Surviving+the+New+Culture+of+Air+Travel\&ots $=$ FvLHlpfT11\&sig=_doEF8ZlsclI6-

soAk6ZLKtZm9c\&redir esc $=\mathrm{y} \# \mathrm{v}=$ onepage $\& \mathrm{q}=$ Life in the Air\%3A Surviving the New Culture of Air Travel\&f=false

[9] John E. Hopcroft, Rajeev. Motwani, and Jeffrey D. Ullman. 2001. Introduction to automata theory, languages, and computation. Addison-Wesley. Retrieved August $31, \quad 2019$ from https://books.google.fi/books/about/Introduction_to_Automata_Theory_Lang uage.html?id=omIPAQAAMAAJ\&redir_esc $=y$

[10] Wei-Jue Huang, Honggen Xiao, and Sha Wang. 2018. Airports as liminal space. Annals of Tourism Research 70: 1-13. https://doi.org/10.1016/j.annals.2018.02.003

[11] Yi Hsin Lin and Ching Fu Chen. 2013. Passengers' shopping motivations and commercial activities at airports - The moderating effects of time pressure and impulse buying tendency. Tourism Management 36: 426-434. https://doi.org/10.1016/j.tourman.2012.09.017

[12] Vincenzo Lombardo and Rossana Damiano. 2012. Storytelling on mobile devices for cultural heritage. New Review of Hypermedia and Multimedia 18, 1-2: 11-35. https://doi.org/10.1080/13614568.2012.617846

[13] Eleni G Mantouka, Emmanouil N Barmpounakis, Christina P Milioti, Eleni G Mantouka, Emmanouil N Barmpounakis, Christina P Milioti, I Eleni, Eleni G Mantouka, Emmanouil N Barmpounakis, Christina P Milioti, and Eleni Vlahogianni. 2018. Gamification in mobile applications: The case of airports Gamification in mobile applications: The case of airports. Journal of Intelligent Transportation Systems $\quad 0, \quad 0$ : $1-10$ https://doi.org/10.1080/15472450.2018.1473157

[14] Lena Mossberg. 2007. A Marketing Approach to the Tourist Experience. Scandinavian Journal of Hospitality and Tourism 7, 1: 59-74. https://doi.org/10.1080/15022250701231915

[15] J Philip, J Ben, Philip J Kirk, Vesna Popovic, and Ben Kraal. 2012. Towards a Taxonomy of Airport Passenger Activities. 1-12.

[16] Meehae Song, Thomas Elias, Ivan Martinovic, Wolfgang Mueller-Wittig, and Tony K Y Chan. 2004. Digital Heritage Application as an Edutainment Tool. $\begin{array}{llll}\text { Retrieved } & \text { September } & 6, & 2018\end{array}$ http://delivery.acm.org/10.1145/1050000/1044621/p163song.pdf?ip $=153.1 .210 .183 \& i d=1044621 \&$ acc $=$ ACTIVE SERVICE\&key=74A0E95D84AAE420.8F78BA8F46D5812F.4D4702B0C3E38B3 5.4D4702B0C3E38B35\&_acm__ 1536228606_46a3d1a998d3160f374c2fa3fa98 $634 \mathrm{a}$

[17] Walanchalee Wattanacharoensil, Markus Schuckert, Anne Graham, and Alison Dean. 2017. Journal of Hospitality and Tourism Management An analysis of the airport experience from an air traveler perspective. Journal of Hospitality and Tourism Management 32: 124-135. https://doi.org/10.1016/j.jhtm.2017.06.003
[18] Walanchalee Wattanacharoensil, Markus Schuckert, Anne Graham, Walanchalee Wattanacharoensil, Markus Schuckert, Anne Graham, Markus Schuckert, and Anne Graham. 2015. An Airport Experience Framework from a Tourism Perspective An Airport Experience Framework from a Tourism Perspective. 1647, October. https://doi.org/10.1080/01441647.2015.1077287

[19] Feifei Xu, Dimitrios Buhalis, and Jessika Weber. 2017. Serious games and the gamification of tourism. Tourism Management 60: 244-256. https://doi.org/10.1016/J.TOURMAN.2016.11.020

[20] Feifei Xu, Jessika Weber, and Dimitrios Buhalis. 2013. Gamification in Tourism. In Information and Communication Technologies in Tourism 2014. Springer International Publishing, Cham, 525-537. https://doi.org/10.1007/978-3-319-03973-2_38

[21] Kamel Ben Youssef, Thomas Leicht, and Lidia Marongiu. 2018. Storytelling in the context of destination marketing: an analysis of conceptualisations and impact measurement. Journal of Strategic Marketing 4488: 1-18. https://doi.org/10.1080/0965254X.2018.1464498

[22] App in the Air - Top mobile app for flight tracking and exploring airports Retrieved June 29, 2018 from https://www.appintheair.mobi/

[23] Helsinki Airport mobile app | Finavia. Retrieved June 29, 2018 from https://www.finavia.fi/en/airports/helsinki-airport/mobile-app

[24] Questionnaire for User Interface Satisfaction. Retrieved July 2, 2018 from http://garyperlman.com/quest/quest.cgi?form=QUIS

[25] USE Questionnaire: Usefulness, Satisfaction, and Ease of use. Retrieved July 2, 2018 from http://garyperlman.com/quest/quest.cgi?form=USE 